\title{
Modulator role of infliximab and methotrexate through the transient receptor potential melastatin 2 (TRPM2) channel in neutrophils of patients with rheumatoid arthritis: a pilot study
}

\author{
Atalay Dogru' ${ }^{1}$, Mustafa Nazıroglu ${ }^{2,3}$, Bilal Cig ${ }^{3}$
}

\begin{abstract}
${ }^{1}$ Department of Internal Medicine, Division of Rheumatology, Dr. Ersin Arslan Training and Research Hospital, Gaziantep, Turkey

${ }^{2}$ Neuroscience Research Center, Suleyman Demirel University, Isparta, Turkey

${ }^{3}$ Department of Biophysics, Faculty of Medicine, Suleyman Demirel University, Isparta, Turkey
\end{abstract}

Submitted: 18 August 2018

Accepted: 29 September 2018

Arch Med Sci 2019; 15 (6): 1415-1424

DOI: https://doi.org/10.5114/aoms.2018.79485

Copyright (c) 2018 Termedia \& Banach

\begin{abstract}
Introduction: Rheumatoid arthritis (RA) is a chronic, systemic, inflammatory disease causing symmetric polyarthritis. In this study, we aimed to investigate the effects of infliximab (INF) and methotrexate (MTX) on apoptosis, oxidative stress, and calcium signaling in the neutrophils of RA patients.

Material and methods: Neutrophils were isolated from 10 patients with newly diagnosed RA and 10 healthy controls. They were divided into four groups (control, RA, RA + MTX, RA + INF) and incubated with MTX and INF. In the cell viability (MTT) test, the ideal non-toxic dose and incubation time of MTX were found to be $0.1 \mathrm{mM}$ and $1 \mathrm{~h}$, respectively. The neutrophils were also incubated with the TRPM2 channel blocker $N$-( $p$-amylcinnamoyl) anthranilic acid (ACA).

Results: Intracellular free $\mathrm{Ca}^{2+}$ concentration, intracellular reactive oxygen species (ROS) production, mitochondrial depolarization, lipid peroxidation, apoptosis, and caspase 3 and caspase 9 activities were found to be significantly higher in the neutrophils of RA patients compared to controls. MTT, reduced glutathione (GSH) level, and glutathione peroxidase (GSHPx) activity were significantly lower in the neutrophils of RA patients. However, MTT, GSH and GSHPx values were detected to be significantly increased with INF and MTX therapies. The $\mathrm{Ca}^{2+}$ concentrations were further decreased by the ACA therapy.

Conclusions: Our results suggest that INF and MTX are useful antagonists in apoptosis and mitochondrial oxidative stress in the neutrophils of RA patients. INF and MTX decreased the $\mathrm{Ca}^{2+}$ concentration through inhibition of the TRPM2 channel in the neutrophils of RA patients. It may be a new pathway in the mechanisms of anti-rheumatic drugs.
\end{abstract}

Key words: apoptosis, oxidative stress, calcium signaling, transient receptor potential melastatin 2 channel, rheumatoid arthritis.

\section{Introduction}

Rheumatoid arthritis (RA) is a chronic, systemic, inflammatory disease causing symmetric polyarthritis (synovitis). Rheumatoid arthritis affects $0.5-1 \%$ of the world population and is most commonly seen between

\author{
Corresponding author: \\ Atalay Dogru MD \\ Department of \\ Internal Medicine \\ Division of Rheumatology \\ Dr. Ersin Arslan \\ Training and \\ Research Hospital \\ 27000 Gaziantep, Turkey \\ Phone: +90 5063698747 \\ E-mail: atalay_dogru@ \\ hotmail.com
}


30 and 50 years of age. Moreover, RA is more common in women than in men. On the other hand, RA is a heterogeneous disease that can be accompanied by extra-articular involvement such as cardiovascular, hematologic, pulmonary, vascular, etc. [1]. The limitation of basic life activities due to joint damage adversely affected the quality of life in RA patients [2]. The primary goal in the treatment of RA is to prevent joint damage by keeping synovitis under control. Methotrexate (MTX) is a disease-modifying antirheumatic drug (DMARD) commonly used in the treatment of RA, which is also known as an anchor drug. However, for patients with DMARD-resistant active RA, tumor necrosis factor $\alpha$ (TNF- $\alpha$ ) inhibitors are an important treatment option [3]. The TNF- $\alpha$ inhibitors have been shown to be effective as monotherapy and combination treatment in randomized studies. Infliximab (INF) is the first monoclonal antibody to human TNF- $\alpha$ developed for RA treatment. INF binds with high affinity to both soluble and trans-membrane TNF and can decrease synovial inflammation, bone resorption, and cartilage degradation [4].

Neutrophils play a key role in the activation, regulation, and effector functions of innate and adaptive immune cells by releasing various cytokines and chemokines. Additionally, neutrophils have a high cytotoxic potential due to their role in the release of destructive enzymes and reactive oxygen species (ROS). When activated improperly, neutrophil extracellular traps (NETs), which are recruited to kill extracellular microorganisms, act as a source of autoantigens. Therefore, neutrophils play a major role in the pathogenesis of diseases such as infections and chronic inflammation in addition to autoimmune diseases [5]. Although there are numerous cells participating in the pathogenesis of RA, neutrophils play the central role among others. As a result, neutrophils from patients with RA often have an increased tendency to generate NETs containing citrullinated proteins that are commonly implicated in the pathophysiology of RA [6].

Oxidative stress (OS) is the impairment in the balance between oxidants and antioxidants in favor of oxidants. OS is known to have a role in the pathogenesis and progression of numerous inflammatory diseases including RA. Moreover, the role of OS in inflammation and joint injury has also been shown in RA patients and in animal studies. Lipid, protein, and DNA oxidation is greater in RA patients compared to healthy individuals, which results in the production of ROS, thereby leading to joint injury $[7,8]$. Transient receptor potential melastatin 2 (TRPM2) is a $\mathrm{Ca}^{2+}$-permeable channel and is abundantly expressed in inflammatory cells including neutrophils. TRPM2 has been shown to act as a sensor for ROS and OS [9]. Increased
OS leads to TRPM2 activation. Additionally, modulation of calcium ion entry through the TRPM2 channel has been shown to decrease apoptosis and OS markers [10]. The MTX and INF therapies are known to decrease OS markers in RA patients [11]. Meaningfully, it is likely that these two drugs, which are highly important for RA treatment, use the TRPM2 channel to exert their effects. The aim of our study was to investigate the effects of MTX and INF on apoptosis, oxidative stress and calcium ion entry through modulation of the TRPM2 channel in the neutrophils of patients with RA. In addition, in light of the findings, we also aimed to shed light on the effect of these two important drugs on the TRPM 2 channel with regards to disease remission.

\section{Material and methods}

\section{Patients and controls}

The study was conducted at the BSN Health Analyses ARGE Ltd., Teknokent, Suleyman Demirel University (SDU) Campus, Isparta, Turkey. The patients enrolled in the study were selected from the patients admitted to SDU Medical School Rheumatology Department. The study was approved by the local ethics committee. The ethics committee approval number was 2017/144. All participants gave written consent, confirming their acceptance for giving blood through the vena brachialis and were informed about the experimental procedures. Demographic characteristics, clinical records, physical examination findings, and laboratory tests were noted for each patient.

The study included 10 patients with newly diagnosed RA and 10 age- and gender-matched control subjects. The diagnosis of RA was established based on the 2010 ACR/EULAR classification criteria for RA [12]. All the patients had active RA. Clinical Disease Activity Index (CDAI), Simple Disease Activity Index (SDAI), Disease Activity Score-28 (DAS-28), useful clinical composite scores that are used for evaluating disease activity in RA were administered to assess disease activity. The visual analog scale (VAS) was also used for assessing disease activity.

Inclusion criteria were as follows: patients who were aged between 18 and 60 years and fulfilled the 2010 ACR/EULAR classification criteria for RA. Exclusion criteria were as follows: concomitant diseases such as diabetes mellitus, hypertension, obesity, hyperlipidemia, depression, autoimmune diseases other than RA, active infection, tobacco or alcohol use, steroid or immunosuppressive treatment within the 4 weeks prior to blood sampling, malignancy or pregnancy, use of vitamin supplements, and previous exposure to biologics or DMARDS. Age- and gender-matched controls 
without any infection and no tobacco, alcohol or drug use were included in the study.

Serum creatinine, erythrocyte sedimentation rate (ESR), C-reactive protein (CRP), rheumatoid factor (RF), cyclic citrullinated peptide antibodies (anti-CCP), uric acid, alanine aminotransferase (ALT), hemogram, and venous blood fasting glucose level (FBG) were measured in each patient after 8-hour overnight fasting. Serum and neutrophil samples were separated from the blood samples. Neutrophil samples were used to measure intracellular $\mathrm{Ca}^{2+}$ concentration $\left(\left[\mathrm{Ca}^{2+}\right] \mathrm{i}\right)$ and were also used for the MTT, apoptosis, intracellular ROS production, mitochondrial depolarization and caspase 3 and 9 activation assays.

\section{Groups}

The neutrophils isolated from the patients and the controls were divided into four groups as follows:

- Control group $(n=10)$ : neutrophils obtained from the healthy subjects were not incubated by INF or MTX.

- RA group ( $n=10)$ : no incubation was performed for the neutrophils obtained from the patients.

- RA + INF $(n=10)$ : neutrophils obtained from the patients were incubated with INF $(0.1 \mathrm{mM})$ for $1 \mathrm{~h} \mathrm{[13].}$

- RA + MTX group $(n=10)$ : Neutrophils obtained from the patients were incubated with MTX $(0.1 \mathrm{mM})$ for $1 \mathrm{~h}$. The dose and the time of MTX incubation were determined using the cell viability (MTT) test.

In the $\mathrm{Ca}^{2+}$ signaling experiments, the effect of TRPM2 on $\mathrm{Ca}^{2+}$ entry was investigated in the neutrophils. To achieve this, the neutrophils in the four groups were further incubated with TRPM2 channel blocker, ACA $(0.025 \mathrm{mM})$, for $10 \mathrm{~min}$ before $\mathrm{fMLP}$ stimulation. Both $\mathrm{AMLP}$ and ACA were purchased from Santa Cruz Inc, (Istanbul, Turkey) and their stock solutions were dissolved in DMSO. Before diluting in extracellular buffer with $\mathrm{Ca}^{2+}$ (1.2 mM), pH adjustment (7.4) was performed both for the agonists and antagonists.

\section{Isolation of human neutrophils}

Neutrophils were isolated from anti-coagulated peripheral blood of the patients and the healthy subjects as described previously [13-15]. Neutrophil isolation was carried out in sterile solutions containing phosphate-buffered saline (PBS) (GIBCO Invitrogen, Istanbul, Turkey), 6\% hydroxyl ethyl starch solution in isotonic $\mathrm{NaCl}$ (Plasmasteril, Fresenius, Bad Homburg, Germany), and Ficoll-PaquePlus Solution (GE Healthcare Bio-Sciences, Uppsala, Sweden). Half of the neutrophils were used for the measurement of $\left[\mathrm{Ca}^{2+}\right] \mathrm{i}, \mathrm{MTT}$, apoptosis, intracellular ROS production, mitochondrial depolarization levels, and caspase 3 and 9 activation. The loading buffer contained HEPES (20 mM), $\mathrm{NaCl}(138 \mathrm{mM}), \mathrm{KCl}(6 \mathrm{mM}), \mathrm{MgCl}_{2}$ (1 $\mathrm{mM}), \mathrm{CaCl}_{2}(1.2 \mathrm{mM})$, and glucose $(5.5 \mathrm{mM})$ with a pH of 7.4. Remaining neutrophils were frozen in the buffer for measuring lipid peroxidation, reduced glutathione (GSH) level, and glutathione peroxidase (GSHPX) activity.

\section{Cell viability (MTT) assay}

Cell viability assays were performed by measuring mitochondrial reductase activity with MTT as described previously $[10,16]$. The neutrophils were incubated in the MTT solution $(0.5 \mathrm{mg} / \mathrm{ml})$ for $15 \mathrm{~min}$. The resulting formazan crystals were dissolved in dimethyl sulfoxide (DMSO; $200 \mu \mathrm{l}$ well). Optical density was measured with a spectrophotometer at 550 and $620 \mathrm{~nm}$ and presented as fold increase over the pretreatment level (experimental/control).

\section{Measurement of $\left[\mathrm{Ca}^{2+}\right] \mathrm{i}$ in neutrophils of patients}

The $\left[\mathrm{Ca}^{2+}\right] \mathrm{i}$ in the neutrophils of patients was measured as described previously $[10,17]$. The neutrophils $\left(1 \times 10^{6}\right.$ neutrophil $\left./ \mathrm{ml}\right)$ were loaded with $2 \mu \mathrm{M}$ Fura-2/AM for $30 \mathrm{~min}$ in the dark at $37^{\circ} \mathrm{C}$ for $45 \mathrm{~min}$. The fMLP was used to stimulate $\mathrm{Ca}^{2+}$ entry. Fluorescence was recorded by a spectrofluorometer (Carry Eclipse; Varian, Sydney, Australia) with excitation wavelengths of 340 and $380 \mathrm{~nm}$ at an emission of $505 \mathrm{~nm}$. Changes in $\left[\mathrm{Ca}^{2+}\right] \mathrm{i}$ were monitored as the fluorescence ratio (Fura-2/AM 340/380 nm). Intracellular calibration for $\mathrm{Ca}^{2+}$ was performed as previously described. $\mathrm{Ca}^{2+}$ entry was estimated using the integral of the rise in $\left[\mathrm{Ca}^{2+}\right]$ i for $170 \mathrm{~s}$ after $\mathrm{fMLP}$ stimulation. $\mathrm{Ca}^{2+}$ release was expressed in nanomoles by noting a reading every second $[13,14]$.

Neutrophils are known to be activated by increased $\left[\mathrm{Ca}^{2+}\right] \mathrm{i}[18]$. Additionally, stimulation of neutrophils by bacterial fMLP is known to induce an increase in $\left[\mathrm{Ca}^{2+}\right] \mathrm{i}$ [19]. Nevertheless, recent reports have shown the modulator role of INF in $\left[\mathrm{Ca}^{2+}\right]$ i through the inhibition of TRPM2 and voltage-gated calcium channels in patients with ankylosing spondylitis. In addition, the modulator role of MTX in oxidative stress in neutrophils stimulated by fMLP has also been reported [20].

\section{Assay of apoptosis and caspase 3 and 9 activities}

For the spectrophotometric analysis of apoptosis, a commercial kit was used and the analyses were performed according to the instructions provided by Biocolor Ltd. (Northern Ireland) and 
elsewhere [17]. In this APOPercentage dye-uptake assay, when the membranes of apoptotic cells loses their asymmetry, the APOPercentage dye is actively transported into the cells, staining apoptotic cells red, thus allowing detection of apoptosis by spectrophotometry.

The determinations of caspase 3 and 9 activities were based on a method previously reported by Kose and Naziroglu [21] with minor modifications. Cleavages of caspase 3 (ACDEVD-AMC) and 9 (AC-LEHD-AMC) substrates were measured with a microplate reader (Infinite pro200; TecanMännedorf, Switzerland) with an excitation wavelength of $360 \mathrm{~nm}$ at an emission of $460 \mathrm{~nm}$. The resulting data were calculated as fluorescence units/mg protein and presented as the fold increase over the pretreatment level (experimental/control).

\section{Intracellular ROS measurement}

Intracellular ROS formation in the neutrophils was spectrofluorometrically estimated using a fluorescent probe, dihydrorhodamine 123 (DHR123), which is oxidized to a fluorescent dye, rhodamine (Rh123), by cellular oxidants, particularly by superoxide radicals [22]. The neutrophils $\left(1 \times 10^{6}\right.$ cells/ $\mathrm{ml}$ ) were first incubated in the presence of $10 \mu \mathrm{M}$ DHR123 for 15 min in incomplete RPMI 1640 medium containing $10 \mathrm{mM}$ HEPES buffer solution at $37^{\circ} \mathrm{C}$. At the end of probe loading, the cells were washed with PBS. The kinetics of RH123 fluorescence intensity (excitation: $488 \mathrm{~nm}$, emission: $530 \mathrm{~nm}$ ) resulting from the oxidation of DHR123 were measured with a plate reader (Infinite pro200).

\section{Measurement of mitochondrial membrane} potential $(\Delta \Psi \mathrm{m})$

JC1 accumulates in the mitochondria depending on the $\Delta \Psi \mathrm{m}$ level and is present either as a monomer or a reversible J-aggregate. The JC1 monomer predominating in depolarized mitochondria emits green fluorescence at $530 \mathrm{~nm}$, whereas the oligomer (J-aggregate) forming in mitochondria with negative potentials emits red fluorescence at $590 \mathrm{~nm}$ [23]. The green (excitation: $485 \mathrm{~nm}$ and emission: $535 \mathrm{~nm}$ ) and red (excitation: $540 \mathrm{~nm}$ and emission: $590 \mathrm{~nm}$ ) JC1 signals were measured in the cell line as described in a previous study [24]. Fluorescence changes were analyzed using a microplate reader (Infinite Pro200). The data were presented as the fold increase over the pretreatment level.

\section{Lipid peroxidation analyses}

Lipid peroxidation in the neutrophils was measured using malondialdehyde (MDA) reaction via the method proposed by Placer et al. [25]. The lipid peroxidation values in the neutrophils were expressed as micromoles per gram of protein. The protein content in the neutrophil samples was measured by the method described by Lowry et al. [26] with bovine serum albumin as the standard.

\section{Reduced glutathione (GSH) and glutathione peroxidase (GSH-Px) assays}

The GSH content of the neutrophil samples was measured spectrophotometrically (UV-1800; Shimadzu, Kyoto, Japan) at $412 \mathrm{~nm}$ according to the method proposed by Sedlak and Lindsay [27]. GSH-Px activity of the neutrophil samples was also measured spectrophotometrically at $37^{\circ} \mathrm{C}$ and $412 \mathrm{~nm}$ according to the method described by Lawrence and Burk [28].

\section{Measurement of non-toxic MTX dosage}

Literature reviews indicate that there is no study in the literature reporting on the dosage and incubation time of MTX in human neutrophils. However, previous studies indicate that in human dendritic cells [29] and intestinal mononuclear cells [30], therapeutic doses of MTX have been used between 0.5 and $5 \mathrm{nM}$. Therefore, in our study, the neutrophils obtained from the healthy controls were incubated with five different doses of MTX $(2,4,6,8$ and $10 \mathrm{nM})$ for $1 \mathrm{~h}$ but no evaluation was performed for the effects of each dosage on the MTT levels (Figure 1 A). Previous studies also indicated that in human chondrocytes, MTX was used up to $100 \mu \mathrm{M}$. Therefore, we increased the dosage of MTX to determine its non-toxic dosage. The neutrophils obtained from the healthy controls were incubated with six different high doses of $\operatorname{MTX}(5,10,20,50,75$ and $100 \mu \mathrm{M})$ for $1 \mathrm{~h}$ (Figure $1 \mathrm{~B}$ ) and we found that the incubation of neutrophils for $1 \mathrm{~h}$ with $0.1 \mathrm{mM}$ MTX was not toxic for the cells (Figure 1).

\section{Biochemical analysis}

The ESR was measured on an Alifax THL 1 ESR analyzer (Alifax SPA, Padua, Italy). Creatinine and ALT were measured using the enzymatic method with a Beckman AU 5800 Autoanalyzer (Beckman Coulter Inc., USA). CRP was measured with a nephelometer (Delta SeacRadim, Pomezia, Italy).

\section{Chemicals}

Roswell Park Memorial Institute (RPMI)-1640 medium, caspase 3 substrate ( $\mathrm{N}$-acetyl-Asp-GluVal-Asp-7-amido-4-methylcoumarin, ACDEVD-AMC), penicillin-streptomycin, 4-(2-hydroxyethyl)-1-piperazineethanesulfonic acid (HEPES), and [3-(4,5-dimethylthiazol-2-yl)-2,5-diphenyltetrazolium bromide] 
A

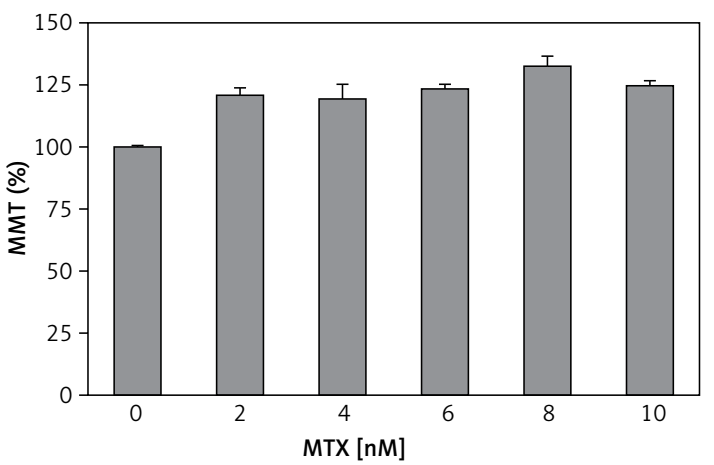

B

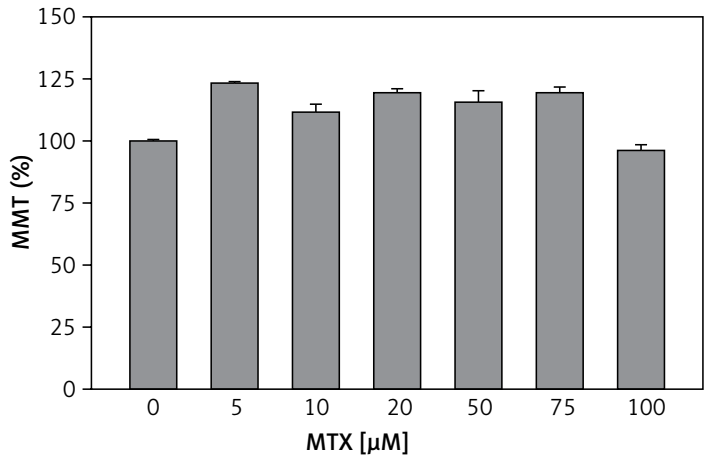

Figure 1. Effects of different doses of MTX on cell viability (MTT) test in the neutrophils of controls (mean \pm SD and $n=3)$. The obtained control neutrophils were incubated in five different low doses $(2,4,6,8$ and $10 \mathrm{nM})(\mathrm{A})$ and six different normal doses $(5,10,20,50,75$ and $100 \mu M)(B)$ of MTX for $1 \mathrm{~h}$. The neutrophils were analyzed by the cell viability (MTT) test. Incubation with $100 \mu \mathrm{M}$ MTX for $1 \mathrm{~h}$ was found to be a nontoxic dosage for the neutrophils

(MTT) were obtained from Sigma Chemical Co. (St. Louis, MO, USA). Dihydrorhodamine-123 (DHR-123), fura-2-acetoxymethyl ester (Fura-2/ AM), 5,5,6,6-tetrachloro-1,1,3,3-tetraethylbenzimidazolylcarbocyanine iodide (JC1), and dimethyl sulfoxide (DMSO) were purchased from Molecular Probes (Eugene, OR, USA). Caspase-9 substrate (Ac-Leu-Glu-His-Asp-AMC, AC-LEHD-AMC) was purchased from Bachem (Bubendorf, Switzerland). $N$-(p-amylcinnamoyl) anthranilic acid (ACA) and fMLP were purchased from Santa Cruz Inc, (Istanbul, Turkey).

\section{Statistical analysis}

Data were analyzed using SPSS for Windows version 17.0 (SPSS Inc. Chicago, Illinois, USA). All the results were expressed as mean \pm standard deviation (SD). Significant values in four groups were determined using the unpaired Mann-Whitney $U$ test. A $p$-value of $<0.05$ was considered significant.

\section{Results}

\section{Demographics and laboratory parameters}

The study included 10 patients with RA (4 men, 6 women) and 10 age-matched healthy subjects. All the patients had active RA. Median age was 46.5 years in the patient group and 40.5 years in the control group. Median body mass index (BMI) was $23.8 \mathrm{~kg} / \mathrm{m}^{2}$ in the patient group and $25.6 \mathrm{~kg} / \mathrm{m}^{2}$ in the control group. Median symptom duration was 7 months in the patient group. No significant difference was found between the two groups with regards to age, gender, $\mathrm{BMI}$, creatinine, and ALT levels. However, ESR and CRP were significantly higher in the patient group than in the control group ( $p=0.001$ for both). In the patient group, median SDAI score was 31.9, median CDAI score was 31.5 and median DAS-28 score was 5.7 (Table I).

\section{Effects of INF and MTX on [ $\left.\mathrm{Ca}^{2+}\right]$ through the inhibition of TRPM 2 channels in neutrophils of patients with RA}

In this study, we aimed to investigate whether the treatment of neutrophils of RA patients with INF and MTX could affect the FMLP-induced $\mathrm{Ca}^{2+}$ mobilization. Figures $2 \mathrm{~A}$ and $\mathrm{B}$ present the effects of INF and MTX on [Ca $\left.{ }^{2+}\right]$ i in the neutrophils of the patients. The $\left[\mathrm{Ca}^{2+}\right] \mathrm{i}$ resulting from $\mathrm{Ca}^{2+}$ entry through TRPM2 channel activation in neutrophils was significantly higher in the patient group compared to the control group ( $p<0.001)$. However, the fMLP-induced $\left[\mathrm{Ca}^{2+}\right] \mathrm{i}$ was significantly decreased in the patients who underwent pre-incubation of neutrophils with INF and MTX $(p<0.001)$. Moreover, [ $\left.\mathrm{Ca}^{2+}\right]$ i was further decreased via combinations of INF + ACA and MTX + ACA and was significantly lower in the INF + ACA and MTX + ACA groups than in the INF and MTX groups, respectively $(p<0.05)$. These findings suggest that the TRPM2 channel is an important channel for $\mathrm{Ca}^{2+}$ entry in the neutrophils of patients with RA.

\section{Effects of INF and MTX on apoptosis, cell viability (MTT), caspase 3 and 9 in neutrophils of patients with RA}

The effects of RA on the programmed apoptosis of neutrophils were evaluated using apoptosis and caspase activation assays. The apoptosis level (Figure $3 \mathrm{~A}$ ) and the caspase 3 and 9 activities (Figure $3 \mathrm{~B}$ ) in the patients with RA were significantly higher compared to the controls although the MTT level was lower in the RA group than in the control group ( $p<0.001$ ) (Figure 3 A). A significant decrease was found in the apoptosis and caspase 3 and 9 activities of the RA patients whose neutrophils were treated with INF and MTX $(p<0.001)$, whereas there was an increase in the MTT level in the INF and MTX groups. These findings suggest that INF and MTX have a protective 
Table I. Age, gender, body mass index (BMI), C-reactive protein (CRP), erythrocyte sedimentation rate (ESR), rheumatoid factor(RF), anti-cyclic citrullinated peptide (anti-CCP), disease activity score-28 (DAS-28); Simplified Disease Activity Index (SDAI), Clinical Disease Activity Index (CDAl), visual analogue scale (VAS) values in controls and RA patients (median ( $25-75$ interquartile ranges) and ${ }^{*} p<0.05$ is significant)

\begin{tabular}{|lccc|}
\hline Parameter & RA $(n=10)$ & Control $(n=10)$ & $P$-value \\
\hline Age $[$ years] & $46.5(34-55.7)$ & $40.5(30-49.2)$ & 0.43 \\
\hline Female, $n(\%)$ & $6(60)$ & $6(60)$ & 1 \\
\hline BMI $\left[\mathrm{kg} / \mathrm{m}^{2}\right]$ & $23.8(21.6-28.2)$ & $25.6(22.5-29.2)$ & $<.63$ \\
\hline CRP $(0-3 \mathrm{mg} / \mathrm{l})$ & $5.2(3-17.5)$ & $1.9(1.3-2.5)$ & $<0.001^{*}$ \\
\hline ESR $[\mathrm{mm} / \mathrm{h}]$ & $29.5(24.2-55)$ & $12(10-18.5)$ & \\
\hline RF & $41(14.5-160)$ & & \\
\hline Anti-CCP & $358(268-500)$ & & \\
\hline DAS-28 & $5.7(5.4-6.3)$ & & \\
\hline SDAl & $31.9(26.1-38.6)$ & & \\
\hline CDAl & $31.5(24.7-38)$ & & \\
\hline VAS & $70(60-80)$ & & \\
\hline
\end{tabular}

A

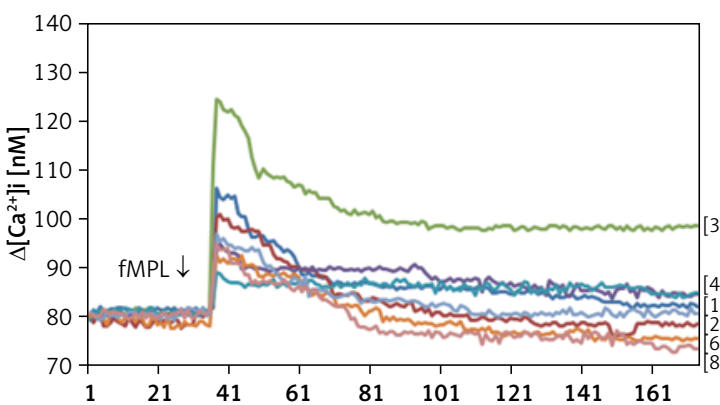

$-[1]$ Control $-[2]$ Control + ACA $-[3]$ RA

$-[4] R A+A C A \quad-[5] R A+I N F X \quad-[6] R A+I N F X+A C A$

$-[7] R A+M T X \quad-[8] R A+M T X+A C A$

\section{B}

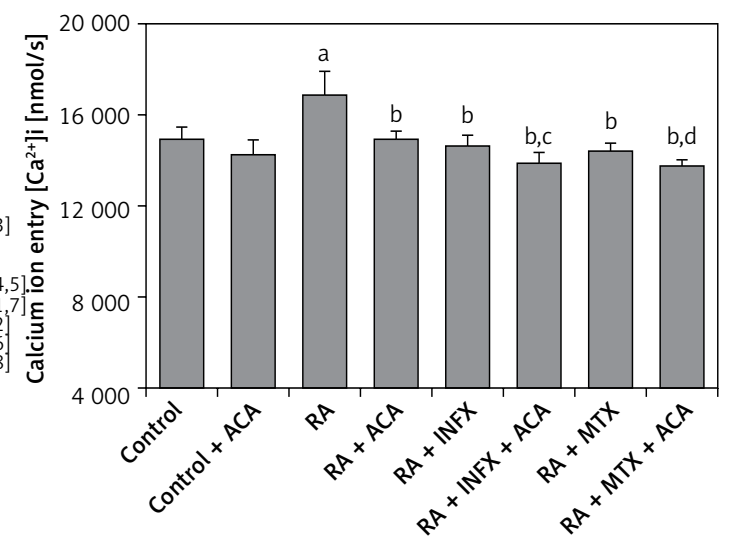

Figure 2. Effect of infliximab (INF) and methotrexate (MTX) incubation on intracellular calcium concentrations (A and B) of the neutrophils of RA patients (mean \pm SD and $n=10$ ). The neutrophils were obtained from both controls and RA patients and were incubated either with infliximab (INF and $0.1 \mathrm{mM}$ for $1 \mathrm{~h}$ ) or infliximab (MTX and $0.1 \mathrm{mM}$ for $1 \mathrm{~h}$ ). Fura-2-loaded neutrophils were stimulated with fMLP $(1 \mu \mathrm{M})$ for $170 \mathrm{~s}$. In some experiments, the neutrophils were incubated with TRPM2 channel blocker, $N$-(p-amylcinnamoyl) anthranilic acid (ACA $0.025 \mathrm{mM}$ for $10 \mathrm{~min})$

${ }^{a} p<0.001$ vs. control. ${ }^{b} p<0.001$ vs. $R A$ group. ${ }^{c} p<0.05$ vs. $R A+I N F$ group. ${ }^{d} p<0.001$ vs. $R A+M T X$ group

A

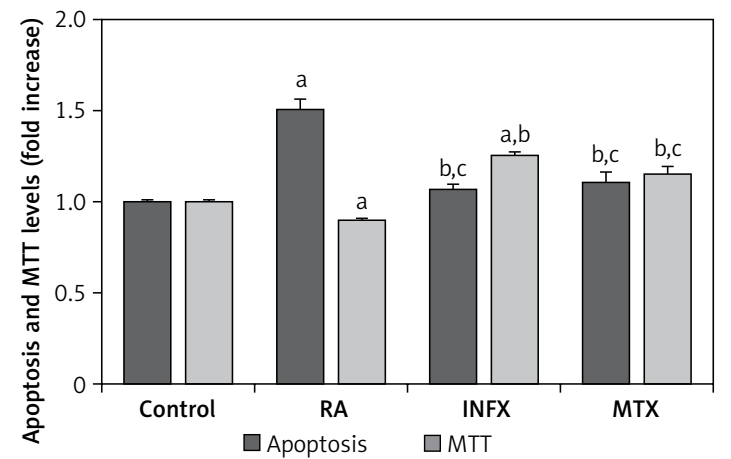

B

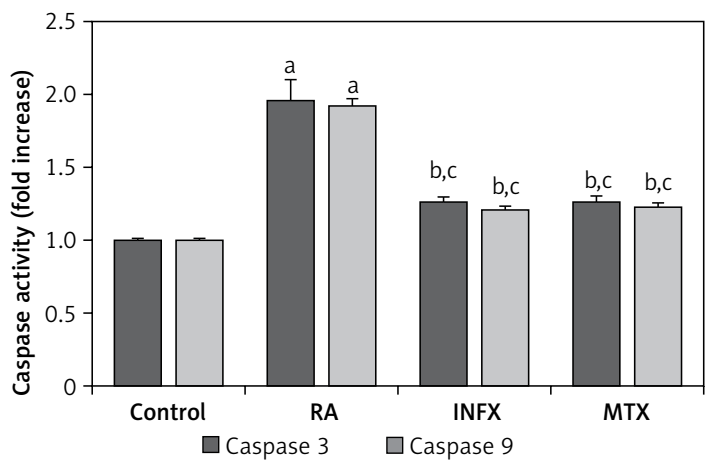

Figure 3. Effect of infliximab (INF) and methotrexate (MTX) incubation on apoptosis, cell viability (MTT) levels (A), caspase 3 and 9 (B) activities in the neutrophils of RA patients (mean \pm SD and $n=10$ )

Values were expressed as fold increase (experimental/control). ${ }^{a} p<0.001$ and ${ }^{c} p<0.05$ vs. control. ${ }^{b} p<0.001$ vs. RA group. 
role in the apoptosis of the neutrophils of RA patients because apoptosis can be inhibited by INF and MTX therapies.

\section{Mitochondrial depolarization (JC1) and intracellular ROS production}

Figure 4 presents the ROS and JC1 results for the RA patients and the healthy controls. Both ROS and JC1 levels were high in the neutrophils of the RA patients compared to healthy controls, whereas the ROS and JC1 levels were significantly lower in the INF and MTX groups as compared to the RA group ( $p \leq 0.001)$.

\section{Lipid peroxidation, GSH, and GSHPx}

Lipid peroxidation, GSH levels, and GSHPX activity were also investigated as indicators of oxidative stress and antioxidant values. Table II presents the lipid peroxidation, GSH, and GSH-Px values. Lipid peroxidation levels were significantly higher in the RA patients compared to the control group and were significantly lower in the INF and MTX groups compared to the control group ( $p<0.05$ for both). In addition, the GSH levels and GSHPx activity were significantly lower in the RA group $(p<0.05)$, whereas a significant recovery was observed in the GSH level and GSH-Px activity in the INF and MTX groups.

\section{Discussion}

In the current study, we evaluated neutrophils since they have cytotoxic and immunoregulatory functions and act as a source of autoantigens. The results indicated that caspase activity, apoptosis, ROS, and JC1 levels were higher in the RA patients compared to healthy controls. Moreover, a significant decrease occurred in these levels and in [ $\left.\mathrm{Ca}^{2+}\right]$ i entry after incubation of the neutrophils with MTX and INF, both of which have been shown in clinical studies to provide effective outcomes in RA treatment.

Calcium signaling cascades play a key role in the activation of immune system cells and in the continuation of immunological events following the activation. $\left[\mathrm{Ca}^{2+}\right] \mathrm{i}$ concentration has been

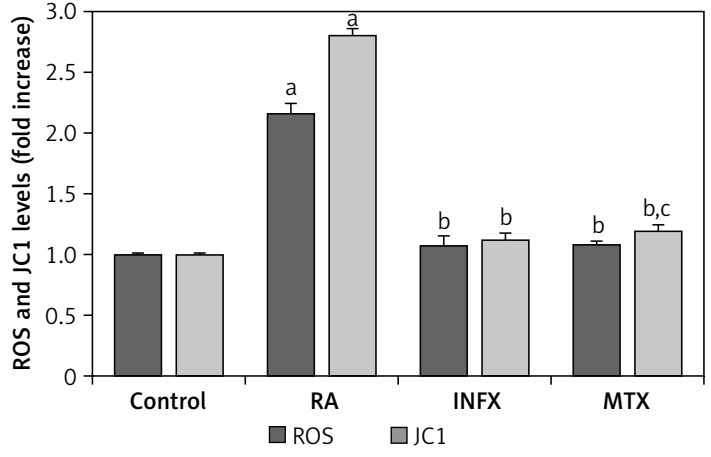

Figure 4. Effect of infliximab (INF) and methotrexate (MTX) incubation on intracellular ROS and mitochondrial membrane depolarization (JC1) levels in the neutrophils of RA patients (mean \pm SD and $n=10$ )

Values were expressed as fold increase (experimental/ control). ${ }^{a} p<0.001$ and ${ }^{c} p<0.05$ vs. control. ${ }^{b} p<0.001$ vs. RA group.

shown to participate in chemotaxis, adhesion, arachnoid acid metabolism, and ROS production [31]. Therefore, some $\mathrm{Ca}^{2+}$ signaling defects have been blamed in the pathogenesis of numerous autoimmune diseases including RA [32]. TRPM2, a member of the TRP family of non-selective cation channels, is a $\mathrm{Ca}^{2+}$-permeable channel embedded in the plasma membrane. TRPM2 also plays a pivotal role in the modulation of basic functions of inflammation including innate immunity response, endothelial barrier integrity, and apoptosis. Additionally, TRPM2 exerts its anti-inflammatory actions via receptor internalization and by preventing neutrophil transmigration through the inactivation of the signal pathway required for the transmigration $[9,33]$. To our knowledge, there has been no study in the literature investigating the role of TRPM2 channel or the efficacy of anti-inflammatory drugs in the neutrophils of RA patients. In our study, intracellular $\left[\mathrm{Ca}^{2+}\right] \mathrm{i}$ concentration through modulation of TRPM 2 channel activation was greater in the neutrophils of RA patients compared to healthy controls. This finding could be related to the etiopathogenesis of RA or disease activation. On the other hand, a significant decrease was observed in $\left[\mathrm{Ca}^{2+}\right]$ i concentration after the incubation of neutrophils with MTX

Table II. Effects of infliximab (INF) and methotrexate (MTX) on lipid peroxidation, reduced glutathione (GSH) and glutathione peroxidase (GSH-Px) values in the neutrophils of controls and RA patients (mean \pm SD and $n=10$ )

\begin{tabular}{|c|c|c|c|c|}
\hline Parameter & Control & RA & $R A+I N F$ & $R A+M T X$ \\
\hline $\begin{array}{l}\text { Lipid peroxidation } \\
\text { [ } \mu \text { mol/g protein] }\end{array}$ & $16.10 \pm 1.61$ & $18.18 \pm 0.54^{a}$ & $15.86 \pm 1.07^{b}$ & $15.65 \pm 0.55^{b}$ \\
\hline $\begin{array}{l}\text { GSH } \\
{[\mu \mathrm{mol} / g \text { protein] }}\end{array}$ & $6.72 \pm 0.32$ & $6.01 \pm 0.30^{a}$ & $6.64 \pm 0.20^{b}$ & $6.67 \pm 0.17^{b}$ \\
\hline $\begin{array}{l}\text { GSH-Px } \\
\text { [IU/g protein] }\end{array}$ & $14.26 \pm 0.66$ & $11.54 \pm 1.57^{a}$ & $13.99 \pm 0.77^{b}$ & $14.32 \pm 1.34^{b}$ \\
\hline
\end{tabular}

${ }^{a} p<0.05$ vs. control group. ${ }^{b} p<0.05$ vs. RA group. 
and INF, although no significant difference was found between the two drugs. This finding implies that although the two drugs provided similar outcomes, they have different effect mechanisms, suggesting that the TRPM 2 channel plays a central role in initiating the inflammatory cascade. Ugan et al. [17] obtained similar findings to our study by evaluating a disease with a different pathogenesis, ankylosing spondylitis, which suggests that the findings obtained in both studies are more associated with the inflammation than the disease. The role of the TRPM 2 channel in inflammation may be greater than thought. Moreover, in our study, the decrease in $\left[\mathrm{Ca}^{2+}\right]$ i concentration became greater when a TRMP2 channel inhibitor (ACA) was added to MTX and INF. TRPM2 channel inhibitors can be used as an adjuvant therapy to standard therapies; however, the resulting molecular response should be clinically supported. Understanding the $\mathrm{Ca}^{2+}$ defects in RA patients and the effect of treatment methods on calcium pathways may lead to the development of novel treatment methods.

Apoptosis is the process of programmed cell death resulting from various physiological pathological conditions. A group of cysteine proteases called 'caspases' are responsible for the collapse of the cell into apoptotic bodies during apoptosis. Caspases exist in cells as inactive proenzymes and are activated by proteolytic cleavage. TNF is a strong inducer of apoptosis that uses caspases. Additionally, it plays a key role in cell death by activating mitochondrial ROS production and c-Jun $\mathrm{N}$-terminal kinase after TNF- $\alpha$ stimulation [34, 35]. Although there are at least 14 caspases, caspase 3 is essential for apoptosis and is triggered by free radicals and lipid peroxidation [36]. Genovese et al. [37] reported that caspase 3 led to a significant reduction in apoptosis-regulating proteins and apoptosis following TNF- $\alpha$ inhibition with INF. Another study reported that the INF therapy led to a significant decrease in caspase 3 activity and apoptotic epithelial loss in patients with Crohn's disease, also noting that the apoptotic epithelial loss that resulted from INF therapy played a key role in the healing of the mucosa [38]. In our study, caspase 3 and 9 activities and apoptosis levels were higher in the neutrophils of RA patients compared to healthy controls and decreased significantly after incubation of the neutrophils with INF. These findings suggest that TNF- $\alpha$ inhibition blocks the apoptotic pathway. Similarly, Shen et al. [39] evaluated a human monocytic cell line and found a significant increase in caspase 3 activity and apoptosis following INF therapy. Weinmann et al. [40] also found that the delayed apoptosis in the neutrophils of RA patients were modulated and increased after MTX therapy. In our study, MTX therapy led to a significant reduction in apoptosis and caspase activity, suggesting that MTX therapy leads to effective suppression of inflammation, thereby normalizing apoptotic pathways.

In patients with RA, ROS production is increased by the activated neutrophils and the ischemia-reperfusion mechanisms of the inflamed joint. Moreover, production of oxidant molecules is increased by disease activation. Modulation of oxidative stress by antioxidant molecules plays a protective role in cartilage and joint damage [7, 41, 42]. Pay et al. [43] reported that the ROS production in neutrophils decreased significantly after the INF therapy. Another study also showed that INF acted as an antioxidant molecule and both oxidative DNA damage and lipid peroxidation decreased after the INF therapy [11]. Similar to INF, MTX has also been shown to result in a significant reduction in oxidant molecules [44-46]. In our study, ROS production, mitochondrial depolarization, and GSH levels were significantly higher in the RA group compared to the control group. Moreover, a significant decrease was observed in the oxidant molecules and a significant increase in the antioxidants. Nevertheless, although no significant difference was observed between the two drugs, both MTX and INF therapies are known to be highly effective and to result in a significant decrease in ROS production, thus shifting the oxidant/antioxidant balance in favor of antioxidants.

Our study was limited in several ways. First, the study had a small patient group and had a cross-sectional design. However, it was determined that the number of patients was sufficient using power analysis before the study started. Another limitation was that other routes of calcium entry, e.g. through membrane voltage/ligand gated calcium channels, were not included in the study. A third limitation is that neutrophil isolation after treatment of patients was not performed and compared with baseline values. The difference between in vitro and in vivo was unknown. Despite these limitations, the inclusion of newly diagnosed and untreated patients in the study is a strength of our study.

In conclusion, MTX and INF, which are two important drugs for RA treatment, reduced calcium ion entry by modulating the TRPM 2 channel. Understanding the role of the TRPM2 channel in RA and the inflammatory cascade and the effect of treatment methods on the calcium pathways may lead to the development of novel treatment methods and new pathways in drug mechanisms. Moreover, MTX and INF led to a remarkable reduction in apoptotic cell death and oxidative stress in RA patients, suggesting that these two drugs may have a role in the modulation of the functions of neutrophils in RA patients. 


\section{Acknowledgments}

There is no support or financial disclosure for the current study. The authors wish to thank the technicians Fatih Sahin and Hulusi Gül (BSN Health Analyses ARGE Ltd., Teknokent, Isparta, Turkey) for their invaluable assistance with plate reader antioxidant analyses.

\section{Conflict of interest}

The authors declare no conflict of interest.

\section{References}

1. Klareskog L, Catrina Al, Paget S. Rheumatoid arthritis. Lancet 2009; 373: 659-72.

2. Baczyk G, Kozlowska K. The role of demographic and clinical variables in assessing the quality of life of outpatients with rheumatoid arthritis. Arch Med Sci 2018; 14: 1070-9.

3. Verstappen SM, Jacobs JW, van der Veen MJ, et al. Intensive treatment with methotrexate in early rheumatoid arthritis: aiming for remission. Computer Assisted Management in Early Rheumatoid Arthritis (CAMERA, an open-label strategy trial). Ann Rheum Dis 2007; 66: 1443-9.

4. Hyrich KL, Symmons DP, Watson KD, Silman AJ; British Society for Rheumatology Biologics Register. Comparison of the response to infliximab or etanercept monotherapy with the response to cotherapy with methotrexate or another disease-modifying antirheumatic drug in patients with rheumatoid arthritis: results from the British Society for Rheumatology Biologics Register. Arthritis Rheum 2006; 54: 1786-94.

5. Thieblemont N, Wright HL, Edwards SW, Witko-Sarsat V. Human neutrophils in auto-immunity. Semin Immunol 2016; 28: 159-73.

6. Wright HL, Moots RJ, Edwards SW. The multifactorial role of neutrophils in rheumatoid arthritis. Nat Rev Rheumatol 2014; 10: 593-601.

7. Mateen S, Moin S, Khan AQ, Zafar A, Fatima N. Increased reactive oxygen species formation and oxidative stress in rheumatoid arthritis. PLoS One 2016; 11: e0152925.

8. Bracht A, Silveira SS, Castro-Ghizoni CV, et al. Oxidative changes in the blood and serum albumin differentiate rats with monoarthritis and polyarthritis. SpringerPlus 2016; 5: 36.

9. Yamamoto S, Shimizu S, Kiyonaka S, et al. TRPM2-mediated $\mathrm{Ca} 2+$ influx induces chemokine production in monocytes that aggravates inflammatory neutrophil infiltration. Nat Med 2008; 14: 738-47.

10. Nazıroğlu M, Sahin M, Ciğ B, Aykur M, Erturan I, Ugan Y. Hypericum perforatum modulates apoptosis and calcium mobilization through voltage-gated and TRPM2 calcium channels in neutrophil of patients with Behcet's disease. J Membr Biol 2014; 247: 253-62.

11. Kageyama Y, Takahashi M, Ichikawa T, Torikai E, Nagano A. Reduction of oxidative stress marker levels by antiTNF-alpha antibody, infliximab, in patients with rheumatoid arthritis. Clin Exp Rheumatol 2008; 26: 73-80.

12. Aletaha D, Neogi T, Silman AJ, et al. 2010 Rheumatoid arthritis classification criteria: an American College of Rheumatology/European League Against Rheumatism collaborative initiative. Arthritis Rheum 2010; 62: 2569-81.

13. Korkmaz S, Erturan I, Nazıroğlu M, Uğuz AC, Ciğ B, Övey IS. Colchicine modulates oxidative stress in serum and neutrophil of patients with Behçet disease through regulation of $\mathrm{Ca}^{2+}$ release and antioxidant system. J Membr Biol 2011; 244: 113-20.

14. Sahin M, Uğuz AC, Demirkan H, Nazıroğlu M. Colchicine modulates oxidative stress in serum and leucocytes from remission patients with family Mediterranean fever through regulation of $\mathrm{Ca}^{2+}$ release and the antioxidant system. J Membr Biol 2011; 240: 55-62.

15. Heiner I, Eisfeld J, Halaszovich CR, et al. Expression profile of the transient receptor potential (TRP) family in neutrophil granulocytes: evidence for currents through long TRP channel 2 induced by ADP-ribose and NAD. Biochem I 2003; 371: 1045-53.

16. Ovey IS, Nazıroglu M. Homocysteine and cytosolic GSH depletion induce apoptosis and oxidative toxicity through cytosolic calciumoverload in the hippocampus og aged mice: involvement of TRPM2 and TRPV1 channels. Neuroscience 2015; 284: 225-33.

17. Ugan Y, Nazıroğlu M, Şahin M, Aykur M. Anti-tumor necrosis factor alpha (Infliximab) attenuates apoptosis, oxidative stress, and calcium ion entry through modulation of cation channels in neutrophils of patients with Ankylosing Spondylitis. J Membr Biol 2016; 249: 437-47.

18. Bréchard S, Tschirhart EJ. Regulation of superoxide production in neutrophils: role of calcium influx. J Leukoc Biol 2008; 84: 1223-37.

19. Heiner I, Eisfeld J, Warnstedt M, Radukina N, Jüngling E, Lückhoff A. Endogenous ADP-ribose enables calciumregulated cation currents through TRPM2 channels in neutrophils granulocytes. Biochem J 2006; 398: 225-32.

20. Okuda A, Kubota M, Watanabe K, et al. Inhibition of superoxide production and chemotaxis by methotrexate in neutrophils primed by TNF-alpha or LPS. Eur J Haematol 1997; 59: 142-7.

21. Köse SA, Nazıroğlu M. N-acetyl cysteine reduces oxidative toxicity, apoptosis, and calcium entry through TRPV1 channels in the neutrophils of patients with polycystic ovary syndrome. Free Radic Res 2015; 49: 338-46.

22. Uğuz AC, Nazıroğlu M, Espino J, et al. Selenium modulates oxidative stress-induced cell apoptosis in human myeloid $\mathrm{HL}-60$ cells via regulation of caspase- $3,-9$ and calcium influx. J Membr Biol 2009; 232: 15-23.

23. Joshi DC, Bakowska JC. Determination of mitochondrial membrane potential and reactive oxygen species in live rat cortical neurons. J Vis Exp 2011; 51: pii: 2704.

24. Espino J, Bejarano I, Paredes SD, et al. Melatonin is able to delay endoplasmic reticulum stress-induced apoptosis in leukocytes from elderly humans. Age (Dordr) 2011; 33: 497-507.

25. Placer ZA, Cushman L, Johnson BC. Estimation of products of lipid peroxidation (malonyldialdehyde) in biological fluids. Anal Biochem 1966; 16: 359-64.

26. Lowry OH, Rosebrough NJ, Farr AL, Randall RJ. Protein measurement with the folin-phenol reagent. J Biol Chem 1951; 193: 265-75.

27. Sedlak J, Lindsay RHC. Estimation of total, protein bound and non-protein sulfhydryl groups in tissue with Ellmann's reagent. Anal Biochem 1968; 25: 192-205.

28. Lawrence RA, Burk RF. Glutathione peroxidase activity in selenium-deficient rat liver. Biochem Biophys Res Commun 1976; 71: 952-58.

29. Kaneno R, Shurin GV, Tourkova IL, Shurin MR. Chemomodulation of human dendritic cell function by antineoplastic agents in low noncytotoxic concentrations. J Transl Med 2009; 7: 58. 
30. Poturoglu S, Kaymakoglu S, GurelPolat N, et al. A new agent for tumour necrosis factor-alpha inhibition: in vitro effects of dipyridamole in Crohn's disease. Scand J Clin Lab Invest 2009; 69: 696-702.

31. Ayub K, Hallett MB. Ca2+ influx shutdown during neutrophil apoptosis: importance and possible mechanism. Immunology 2004; 111: 8-12.

32. Hemon P, Renaudineau $Y$, Debant $M$, et al. Calcium signaling: from normal $B$ cell development to tolerance breakdown and autoimmunity. Clin Rev Allergy Immunol 2017; 53: 141-65.

33. Wang G, Cao L, Liu X, et al. Oxidant sensing by TRPM2 inhibits neutrophil migration and mitigates inflammation. Dev Cell 2016; 38: 453-62.

34. Rath PC, Aggarwal BB. TNF-induced signaling in apoptosis. J Clin Immunol 1999; 19: 350-64.

35. Kim JJ, Lee SB, Park JK, Yoo YD. TNF-alpha-induced ROS production triggering apoptosis is directly linked to Romo1 and Bcl-XL. Cell Death Differ 2010; 17: 1420-34.

36. Abogresha NM, Greish SM, Abdelaziz EZ, Khalil WF. Remote effect of kidney ischemia-reperfusion injury on pancreas: role of oxidative stress and mitochondrial apoptosis. Arch Med Sci 2016; 12: 252-62.

37. Genovese T, Mazzon E, Crisafulli C, et al. TNF-alpha blockage in a mouse model of $\mathrm{SCl}$ : evidence for improved outcome. Shock 2008; 29: 32-41.

38. Eder P, Lykowska-Szuber L, Krela-Kazmierczak I, Stawczyk-Eder K, Zabel M, Linke K. The influence of infliximab and adalimumab on the expression of apoptosis-related proteins in lamina propria mononuclear cells and enterocytes in Crohn's disease - an immunohistochemical study. J Crohns Colitis 2013; 7: 706-16.

39. Shen C, Van Assche G, Rutgeerts P, Ceuppens JL. Caspase activation and apoptosis induction by adalimumab: demonstration in vitro and in vivo in a chimeric mouse model. Inflamm Bowel Dis 2006; 12: 22-8.

40. Weinmann P, Moura RA, Caetano-Lopes JR, et al. Delayed neutrophil apoptosis in very early rheumatoid arthritis patients is abrogated by methotrexate therapy. Clin Exp Rheumatol 2007; 25: 885-7.

41. Hassan SZ, Gheita TA, Kenawy SA, Fahim AT, El-Sorougy IM, Abdou MS. Oxidative stress in systemic lupus erythematosus and rheumatoid arthritis patients: relationship to disease manifestations and activity. Int J Rheum Dis 2011; 14: 325-31.

42. Qiao YQ, Jiang PF, Gao YZ. Lutein prevents osteoarthritis through Nrf2 activation and downregulation of inflammation. Arch Med Sci 2018; 14: 617-24.

43. Pay S, Musabak $\mathrm{U}$, Erdem $\mathrm{H}$, et al. Chimerical antiTNF-alpha, infliximab, inhibits neutrophil chemotaxis and production of reactive oxygen species by blocking the priming effect of mononuclear cells on neutrophils. Immunopharmacol Immunotoxicol 2005, 27: 187-98.

44. Laurindo IM, Mello SB, Cossermelli W. Influence of low doses of methotrexate on superoxide anion production by polymorphonuclear leukocytes from patients with rheumatoid arthritis. J Rheumatol 1995; 22: 633-8.

45. Sung JY, Hong JH, Kang HS, et al. Methotrexate suppresses the interleukin- 6 induced generation of reactive oxygen species in the synoviocytes of rheumatoid arthritis. Immunopharmacol 2000; 47: 35-44.

46. Zimmerman MC, Clemens DL, Duryee MJ, et al. Direct antioxidant properties of methotrexate: inhibition of malondialdehyde-acetaldehyde-protein adduct formation and superoxide scavenging. Redox Biol 2017; 13 588-93. 